\title{
Single Crystal Growth, Transport, and Electronic Band Structure of YCoGa5
}

\author{
Xiangde Zhu ${ }^{\mathrm{a}, *}$, Wenjian Lu ${ }^{\mathrm{b}, *}$, Wei Ning ${ }^{\mathrm{a}}$, Zhe $\mathrm{Qu}^{\mathrm{a}}$, Li Lic ${ }^{\mathrm{c}}$, T. F. Qi ${ }^{\mathrm{c}}$, Gang \\ $\mathrm{Cao}^{\mathrm{c}}$, Cedomir Petrovic ${ }^{\mathrm{d}}$, Yuheng Zhang ${ }^{\mathrm{a}}$ \\ ${ }^{a}$ High Magnetic Field Laboratory, Chinese Academy of Sciences, Hefei 230031, China \\ ${ }^{b}$ Key Laboratory of Materials Physics, Institute of Solid State Physics, Chinese Academy \\ of Sciences, Hefei 230031, China \\ ${ }^{c}$ Department of Physics and Astronomy and Center for Advanced Materials, University \\ of Kentucky, Lexington, KY 40506, USA \\ ${ }^{d}$ Condensed Matter Physics and Materials Science Department, Brookhaven National \\ Laboratory, Upton, NY 11973, USA
}

\begin{abstract}
Single crystal of $\mathrm{YCoGa}_{5}$ has been grown via Ga self-flux. In this paper, we report the single crystal growth, crystallographic parameters, resistivity, heat capacity, and band structure results of YCoGa. YCoGas accommodates the HoCoGa5 type structure (space group P4/mmm (No. 123), Z = $1, \mathrm{a}=4.2131(6) \AA, \mathrm{c}=6.7929(13) \AA$, which is isostructural to the extensively studied heavy fermion superconductor system $\mathrm{Ce} M \operatorname{In}_{5}(M=\mathrm{Co}, \mathrm{Rh}$, Ir) and the unconventional superconductor $\mathrm{PuCoGa}_{5}$ with $T_{c}=18.5 \mathrm{~K}$. No superconductivity is observed down to $1.75 \mathrm{~K}$. Band structure calculation results show that its band at the Fermi level is mainly composed of Co-3d and Ga-4p electrons states, which explains its similarity of physical properties to $\mathrm{YbCoGa}_{5}$ and $\mathrm{LuCoGa}_{5}$.
\end{abstract}

Keywords: A. intermetallics; B. crystal growth; C. crystal structure; C. electrical transport.

*Corresponding authors at: High Magnetic Field Laboratory, Chinese Academy of Sciences, Hefei 230031, China. TEl: +86-551-65595619; FAX: +86-551-65591149. Email: xdzhu@hmfl.ac.cn(Experiment);wjlu@issp.ac.cn(Calculation) 


\section{Introduction}

New materials may stimulate extensive research, and could clarify or bring forth important questions about the underlying physics. For example, $\mathrm{CeCoIn}_{5}$, the heavy fermion superconductor with the highest superconducting transition temperature $\left(T_{c}\right)$ of $2.3 \mathrm{~K}$, was discovered by Petrovic et al. in 2001. It became the most extensively studied heavy fermion system during the past decade.[1] Later in 2002, Sarrao et al. reported the discovery of $5 f$ superconductor $\mathrm{PuCoGa}_{5}$ with $T_{c}=18.5 \mathrm{~K}$.[2] These two compounds strongly support the unconventional superconductivity mediated by magnetic fluctuations. [3] Both of them belong to the ternary HoCoGa 5 -type tetragonal structure (space group $\mathrm{P} 4 / \mathrm{mmm}$ ), which can be regarded as alternative stacking of $\mathrm{HoGa}_{3}-\mathrm{CoGa}_{2}-\mathrm{HoGa}_{3}$ layers sequentially along the $c$-axis. This leads to rather unique and quasi two dimensional electronic states. Due to the heavy fermion superconductor $\mathrm{CeMIn}_{5}$ ( $\mathrm{M}$ is $\mathrm{Co}, \mathrm{Rh}, \mathrm{Ir}$ ) materials and the wide family of $L n \mathrm{TX}_{5}$ and $A n \mathrm{TX}_{5}$ (here, $L n$ is the rare earth atoms, $A n$ is the actinide, $\mathrm{T}$ is transition metal, $\mathrm{X}$ is $\mathrm{In}$ or $\mathrm{Ga}$ ), [4, 5, [6, 6, 7, 8] the HoCoGa $\mathrm{H}_{5}$ type structure have attracted attention both from physics and chemistry fields. So far, only Gd, Tb, Dy, Ho, Er, Tm, Yb, Lu and Y can form HoCoGa 5 type strucutre. [8] The single crystals for Tb-Tm were grown by Hudis et al, showing metallic behavior with Kondo antiferromagnetism. [6] The single crystal for $\mathrm{Yb}\left(4 \mathrm{f}^{14} 6 \mathrm{~S}^{2}\right) \mathrm{CoGa}_{5}$ and $\mathrm{Lu}\left(4 \mathrm{f}^{14} 5 \mathrm{~d}^{1} 6 \mathrm{~S}^{2}\right) \mathrm{CoGa}_{5}$ were grown by Okudzeto et al. [9] and Matsuda et al.[10], respectively. Although Yb usually shows valence fluctuations, spin fluctuations and quantum critical behavior in many Yb-based intermetallic compound, [11, 12, 13, 14, 15, 16, 17] YbCoGa 5 shows typical simple metallic behavior similar to $\mathrm{LuCoGa}_{5} .[9$, 10] Thus, Yttrium without f electrons may provide a good reference to study structure-property relations in $115 \mathrm{Ga}$ compounds. So far, we have noticed that YCoGas has no report on single crystal or detailed physical properties in our knowledge, except for the structure report. [8].

In addition, 13 phase compound $\mathrm{CeIn}_{3}$ (space group $\operatorname{Pr} \overline{3} m, \mathrm{a}=4.689 \AA$ ) has also attracted some attentions due to its tunable superconductivity and antiferromagnetism under pressure. 18] The iso-structural $\mathrm{ScGa}_{3}$ and $\mathrm{LuGa}_{3}$ are both type I superconductors.[19] Unfortunately, $\mathrm{YGa}_{3}$ does not exist. Thus, it is interesting to search for the superconductivity in the $\mathrm{YCoGa}_{5}$, since its structure is composed of $\mathrm{YGa}_{3}-\mathrm{CoGa}_{2}-\mathrm{YGa}_{3}$ layers. In this paper, we report single crystal growth, crystallographic parameters, physical properties and electronic structure of $\mathrm{YCoGa}_{5}$. 


\section{Material and methods}

Single Crystal Growth. The single crystal of YCoGas was grown via gallium self-flux method. Yttrium shot (Alfa Aesar, 99.9\%), Cobalt powder (Aladin, 99.9\%), and Gallium shot (99.995\%) in 1:1:30 mol ratio were put in an $2 \mathrm{~mL}$ aluminum crucible, and sealed in an evacuated quartz tube. The tube was heated to $1100{ }^{\circ} \mathrm{C}$, and dwelled for $6 \mathrm{~h}$, then cooled down to $400{ }^{\circ} \mathrm{C}$ in a $2 \mathrm{~K} / \mathrm{h}$ rate. At this temperature, crystals were separated from the flux using a centrifuge. Single crystal with shiny face can be found with the size of $5 \times 5 \times 3 \mathrm{~mm}^{3}$, which is shown in Fig. 2. In order to remove the Ga flux on the surface, the sample was put in boiling water for about 30 minutes, and then the etching in dilute $\mathrm{HCl}$ for several hours.

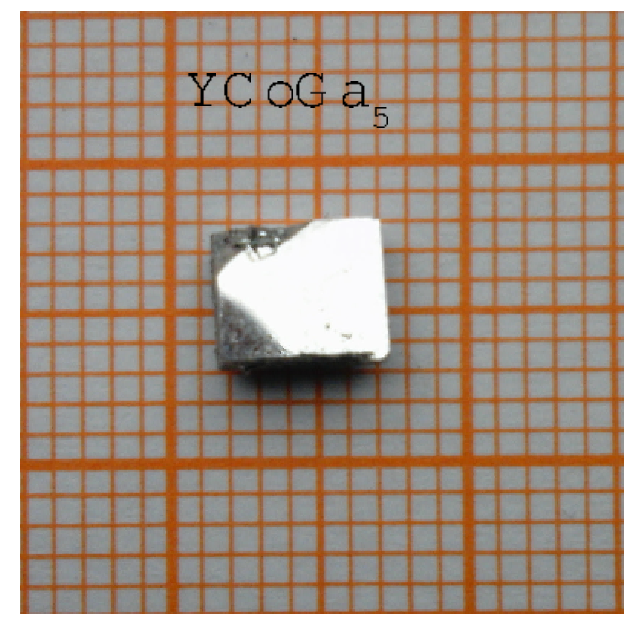

Figure 1: The photograph of as-grown YCoGa5 single crystal.

Single X-ray Diffraction and Elemental Analysis. Crystal fragments with dimensions of about $0.1 \times 0.1 \times 0.1 \mathrm{~mm}^{3}$ were mechanically cut from the crystal and selected for the single crystal X-ray experiments of $\mathrm{YCoGa}_{5}$. The crystal was glued on the tip of a quartz fiber and mounted on a Nonius Kappa CCD diffractometer outfitted with a Mo Kappa radiation $(\lambda$ )$=0.71073 \AA)$. Data collection was taken at $296 \mathrm{~K}$. The crystal structure of YCoGa 5 was solved using SHELXS97 and refined using SHELXL97. [20] The crystallographic parameters are listed in Table 1. After refinement the data were corrected for extinction effects and the displacement parameters were refined as anisotropic. A list of atomic positions, Wyckoff symmetry, and anisotropic displacement parameters are shown in Table 1. The composition 
of the single crystal was determined by energy dispersive X-ray spectrum (EDS) on Hitachi TM3000 Tabletop Microscope system. The average elemental ratio is $\mathrm{Yb} / \mathrm{Co} / \mathrm{Ga} \sim 1.1: 1: 4.93$.

Transport Properties. Electrical transport measurement was carried out on a Linear Research AC bridge and a Quantum Design Magnetism Property Measurement System, with the current applied in the $a b$-plane of the sample. Data were collected over a temperature range of $1.75-300$ K. Temperature dependent resistivity measurement was performed using a four-probe configuration. Gold wires were attached to a polished bar-shaped sample with the electrical contacts made by silver epoxy. Heat capacity measurement was carried out on a Quantum Design Physical Property Measurement System PPMS-16.

Band Calculations. The electronic strucutre of $\mathrm{YCoGa}_{5}$ were calculated by first principles within density functional theory (DFT). The calculations were performed by means of the projected augmented-wave (PAW) [21, 22] formalism implemented in the ABINIT code. [23, 24, 25] The exchangecorrelation functions are treated by using the generalized gradient approximation (GGA) according to the Perdew-Burke-Ernzerhof (PBE) [26] parametrization. The basis set of valence electronic states was taken to be $4 s^{2} 4 p^{6} 4 d^{1} 5 s^{2}$ for Y, $3 s^{2} 3 p^{6} 3 d^{7} 4 s^{2}$ for Co, and $3 s^{2} 3 p^{6} 3 d^{10} 4 s^{2} 4 p^{1}$ for Ga, respectively. Electronic wave functions are expanded with plane waves up to an energy cutoff $\left(E_{\text {cut }}\right)$ of $1400 \mathrm{eV}$. Brillouin zone sampling is performed on a Monkhorst-Pack (MP) mesh [27] of $16 \times 16 \times 12$. The self-consistent calculations were considered to be converged when the total energy of the system was stable within $10^{-6} \mathrm{eV}$ per atom.

\section{Results and Discussions}

\subsection{Crystal Structure}

As shown in Fig. 2(a), the crystal structure of $\mathrm{YCoGa}_{5}$ is composed of alternative $\mathrm{YGa}_{3}$ cuboctahedra layers and $\mathrm{CoGa}_{2}$ rectangular prisms layers stacking along c-axis. Figure 2 (b), (c), (d) and (e) shows (0kl), (h0l), (hk0) and (hk3) reciprocal planes from the single crystal X-ray diffraction patterns at $T=296 \mathrm{~K}$, respectively. Obviously, the well-defined patterns indicate that the single crystal is of high quality. The crystallographic and atom parameters for YCoGa $\mathrm{Y}_{5}$ obtained from Rietveld Refinement are listed in Tab. 1. The lattice parameters are consistent with the previous report. All the 
parameters for $\mathrm{YCoGa}_{5}$ are very close to those for $\mathrm{YbCoGa}_{5}$ and $\mathrm{LuCoGa}_{5} \cdot 9$, 10]
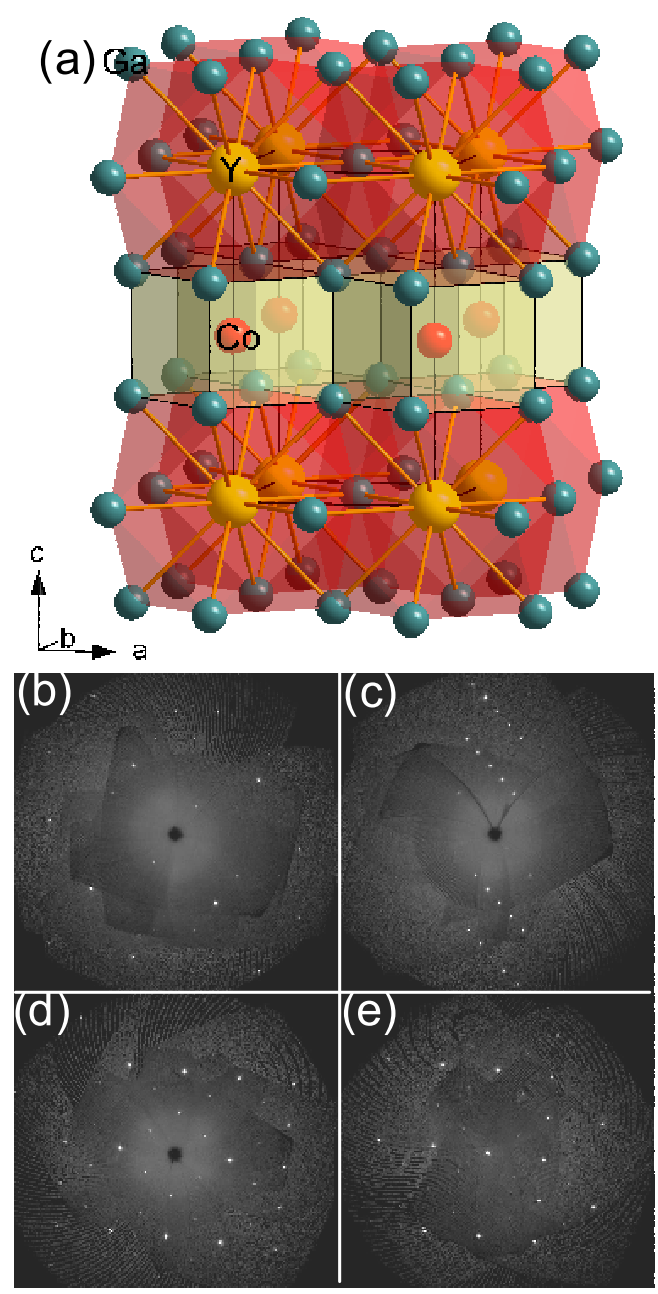

Figure 2: (a) The crystal structure of YCoGa. (b), (c), (d), (e) shows (0kl), (h0l), (hk0), (hk3) reciprocal planes from the single crystal X-ray diffraction pattern at $\mathrm{T}=296 \mathrm{~K}$, respectively.

\subsection{Transport Properties}

Figure 3(a) shows the temperature dependence of in-plane resistivity $\left(\rho_{a b}\right)$ for $\mathrm{YCoGa}_{5}$. Obviously, it shows a typical metallic behavior, with a small residual resistivity $\left(\rho_{0}\right)$ of $0.14 \mu \Omega \mathrm{cm}$. The obtained residual resistivity ratio 
Table 1: Crystallographic parameters for $\mathrm{YCoGa}_{5}$.

\begin{tabular}{|c|c|}
\hline Parameters & $\mathrm{YCoGa}_{5}$ \\
\hline S.G. & $\mathrm{P} 4 / m m m(123)$ \\
\hline $\mathrm{a}$ & $4.2131(6) \AA$ \\
\hline $\mathrm{c}$ & $6.7929(14) \AA$ \\
\hline $\mathrm{c} / \mathrm{a}$ & 1.612 \\
\hline $\mathrm{V}\left(\AA^{3}\right)$ & $120.58(3)$ \\
\hline $\mathrm{Z}$ & 1 \\
\hline dimensions (mm) & $0.1 \times 0.1 \times 0.1$ \\
\hline Density & $6.837 \mathrm{~g} \mathrm{~cm}^{-3}$ \\
\hline Temperature & $296 \mathrm{~K}$ \\
\hline$\theta$ range & $3-27.4^{\circ}$ \\
\hline collected reflections & 114 \\
\hline$h$ & $-3 \leq h \leq 3$ \\
\hline$k$ & $-5 \leq k \leq 5$ \\
\hline$l$ & $-8 \leq l \leq 8$ \\
\hline$\Delta \rho_{\max }\left(\mathrm{e} \AA^{-3}\right)$ & 2.70 \\
\hline$\Delta \rho_{\min }\left(\mathrm{e} \AA^{-3}\right)$ & -1.54 \\
\hline${ }^{a} R_{1}\left[\mathrm{~F}^{2}>2 \sigma\left(\mathrm{F}^{2}\right)\right]$ & 0.035 \\
\hline${ }^{b} R_{w}$ & 0.109 \\
\hline \multicolumn{2}{|c|}{$\begin{array}{l}\mathrm{a} \\
R_{1}=\sum|| F_{o}|-| F_{c}|| / \sum\left|F_{0}\right| . \\
\mathrm{b} \\
\left.R_{w}=\left[\sum\left[w\left(F_{o}^{2}-F_{c}^{2}\right) 2\right] / \sum\left[w\left(F_{o}^{2}\right)^{2}\right]\right]\right]^{1 / 2} ; \\
w=1 /\left[\sigma^{2}\left(F_{o}^{2}\right)+(0.065 P)^{2}+0.5173 P\right] ; P \\
\quad=\left(F_{o}^{2}+2 F_{c}^{2}\right) / 3 .\end{array}$} \\
\hline
\end{tabular}

$(\mathrm{RRR})$ is $\sim 62$, indicating the high quality of the single crystal. These values are comparable with those of $\mathrm{YbCoGa}_{5}$ and $\mathrm{LuCoGa}_{5} \cdot[9$, 10] As shown in Fig. 3(b), the linear relationship of $\rho(T)-T^{3}$ can be observed, rather than $\rho(T)-T^{5}$ for usual electron-phonon scattering. This demonstrates that the $s-d$ electron scattering is the major scattering mechanism in YCoGa. $\mathrm{Ys}_{5}$ shown in Fig. 3(a), $\rho_{a b}-T$ curve can be fitted with the Bloch-Grüneisen formula with $n \sim 3$

$$
\rho(T)=\rho_{0}+2 A_{a c}\left(\frac{T}{\Theta_{R}}\right)^{n-1} T \int_{0}^{\frac{\Theta_{R}}{T}} \frac{x^{n}}{\left(e^{x}-1\right)\left(1-e^{-x}\right)} d x
$$

in the whole range, where $\Theta_{R}$ is the Debye temperature obtained from the resistivity measurements, $A_{a c}$ is the temperature dependent coefficient. The 
Table 2: The fractional coordinates and thermal parameters for YbCoGa5. Here, $U_{e q}$ is defined as $1 / 3$ of the trace of the orthogonalized $U_{i j}$ tensor.

\begin{tabular}{cccccc}
\hline atom & Wyckoff & $\mathrm{x}$ & $\mathrm{y}$ & $\mathrm{z}$ & $U_{e q}$ \\
\hline $\mathrm{Y}$ & $1 \mathrm{a}$ & 0 & 0 & 0 & $0.0011(7)$ \\
$\mathrm{Co}$ & $1 \mathrm{~b}$ & 0 & 0 & $1 / 2$ & $0.0029(7)$ \\
$\mathrm{Ga} 1$ & $1 \mathrm{c}$ & $1 / 2$ & $1 / 2$ & 0 & $0.0112(7)$ \\
$\mathrm{Ga} 2$ & $4 \mathrm{i}$ & 0 & $1 / 2$ & $0.3107(2)$ & $0.0118(6)$
\end{tabular}

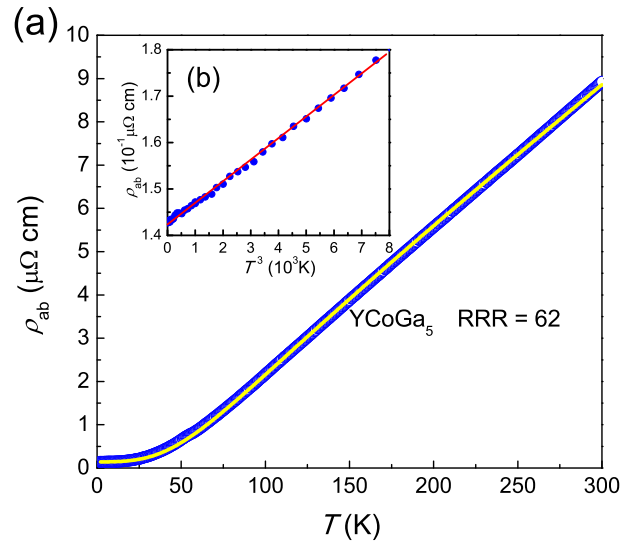

Figure 3: (a) The temperature dependence of in-plane resistivity $\left(\rho_{a b}\right.$, circle) and its fitting results (solid line) for $\mathrm{YCoGa}_{5}$. (b) The $\rho_{a b}$ versus $T^{3}$ at low temperature for YCoGa . The solid line serves as a guide of eye. 


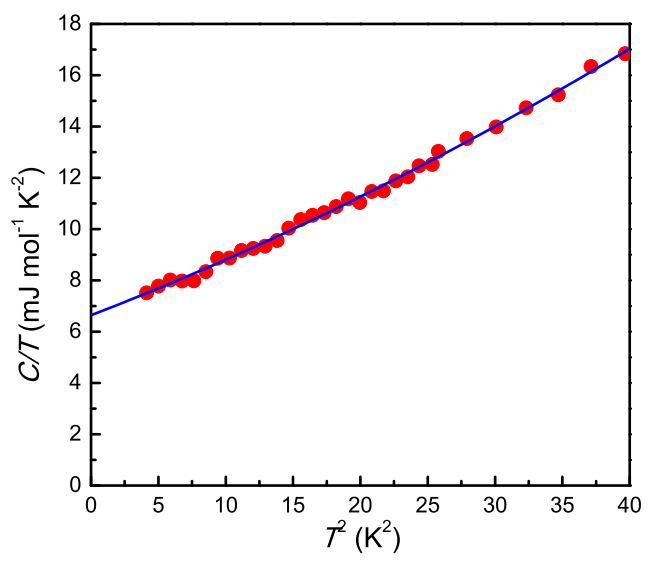

Figure 4: The $T^{2}$ dependence of specific heat $(C)$ divided by $T$ for $\mathrm{YCoGa}_{5}$. The solid line shows the fitting results.

fitting gives $A_{a c}=0.061223 \mu \Omega \mathrm{cm} \mathrm{K}^{-1}$, and $\Theta_{R}=338 \mathrm{~K}$. This type of $\rho-T$ relation has been observed in $\mathrm{LuIn}_{3}, 28$ and $\mathrm{LuGa}_{3}$. 19.

Figure 4 depicts the $T^{2}$ dependence of specific heat $(C)$ divided by $T$ for YCoGa 5 . The data can be well fitted by the formula $C=\gamma T+\beta T^{3}+\delta T^{5}$, where $\gamma T$ is the electron contribution, $\beta T^{3}$ is the phonon contribution, and $\delta T^{5}$ is the anharmonic contribution. The obtained results from the fitting gives $\gamma=6.64 \pm 0.11 \mathrm{~mJ} \mathrm{~mol}^{-1} \mathrm{~K}^{-2}, \beta=0.202 \pm 0.012 \mathrm{~mJ} \mathrm{~mol}^{-1} \mathrm{~K}^{-4}$, and $\delta=1.4 \pm 0.3 \mathrm{~mJ} \mathrm{~mol}^{-1} \mathrm{~K}^{-6} \cdot \Theta_{D}=407 \pm 8 \mathrm{~K}$ is estimated from the $\Theta_{D}=\sqrt[3]{12 \pi^{4} N R / 5 \beta}$, where $\mathrm{N}$ is the number of atoms per molecule.

All the lattice and physical parameters for single crystal $\mathrm{YCoGa}_{5}, \mathrm{YbCoGa}_{5}$, $\mathrm{LuCoGa}_{5}$ and $\mathrm{PuCoGa}_{5}$ are listed in Tab. 3. The lattice parameters and the $c / a$ ratios are very close to each other, as well as the physical parameters except $\mathrm{PuCoGa}_{5}$. Therefore, it is worthwhile to figure out the physics origin of the similarity between $\mathrm{YCoGa}_{5}, \mathrm{YbCoGa}_{5}$ and $\mathrm{LuCoGa}_{5}$. Band structure calculation will provide good opportunity to understand their physical properties.

\subsection{Band Calculations}

The theoretically optimized lattice parameters of $\mathrm{YCoGa}_{5}$ are established to be $a=4.227 \AA$ and $c=6.805 \AA$, which are very close to the experimental values. The band dispersion is shown in the supporting information. The calculated total and projected density of states (DOS) are shown in Fig. 5 . 
Table 3: The lattice and physical parameters for single crystal YCoGa $a_{5}, Y_{6 C o G a}$, $\mathrm{LuCoGa}_{5}$ and $\mathrm{PuCoGa}_{5}$.

\begin{tabular}{|c|c|c|c|c|}
\hline & $\mathrm{YCoGa}_{5}$ & $\mathrm{YbCoGa}_{5}$ & $\mathrm{LuCoGa}_{5}$ & $\mathrm{PuCoGa}_{5}$ \\
\hline $\mathrm{a}(\AA)$ & $4.2131(6)$ & $4.190(1)$ & $4.184(3)$ & 4.232 \\
\hline$c(\AA)$ & $6.7929(14)$ & $6.727(3)$ & $6.718(7)$ & 6.786 \\
\hline $\mathrm{c} / \mathrm{a}$ & $1.6122(6)$ & $1.605(6)$ & $1.606(3)$ & 1.603 \\
\hline$\rho_{0}(\mu \Omega \mathrm{cm})$ & 0.14 & 0.13 & 0.31 & $\sim 35$ \\
\hline RRR & 62 & 150 & 52 & $\sim 7$ \\
\hline$\gamma\left(\mathrm{mJ} \mathrm{mol}^{-1} \mathrm{~K}^{-2}\right)$ & 6.64 & 11.2 & $\sim 6$ & 77 \\
\hline$\Theta_{D}(\mathrm{~K})$ & 408 & $420^{a}$ & $344^{a}$ & \\
\hline Referece & This work & $\operatorname{Ref}[9]$ & $\operatorname{Ref}[10]$ & $\operatorname{Ref}[2]$ \\
\hline
\end{tabular}

The DOS in the region from $-10 \mathrm{eV}$ to $-4 \mathrm{eV}$ mainly consist of Ga- $4 s$ states, which are far from the Fermi level $E_{F}$. There are two peaks below $E_{F}$ due to Co- $3 d$ states that strongly hybridize with Ga- $4 p$ states between $-4 \mathrm{eV}$ and $3 \mathrm{eV}$ in valence and conduction bands. Due to the hybridization, the bandwidth of Co-3d states becomes wide and results in an itinerant metallic behavior for $\mathrm{YCoGa}_{5}$. As shown in Fig. 5, the DOS at Fermi level $N\left(E_{F}\right)$ mainly consist of Co- $3 d$ and Ga- $4 p$ states, while Y- $4 d$ state palys a minor role; and the calculated value of $N\left(E_{F}\right)$ is 1.86 states/eV. In addition, the $E_{F}$ nearly locates at a local minimum in the total DOS, suggesting a higher structure stability of $\mathrm{YCoGa}_{5}$, since it signifies a barrier for electrons below $E_{F}$ to move into unoccupied empty states.

As is shown in Tab. 3, YCoGa, $\mathrm{YbCoGa}_{5}$ and $\mathrm{LuCoGa}_{5}$ have similar lattice parameters. All of them show normal metallic behavior with small residual resistivity, non-magnetism, and non-superconductivity. For LuCoGa5, the DOS at Fermi level $N\left(E_{F}\right)$ mainly consist of Co-3d and Ga- $4 p$ states, jus as the same as $\mathrm{YCoGa}_{5}$.[10] This strongly suggests that $\mathrm{Yb}$ should be divalent in $\mathrm{YbCoGa}_{5}$ ( $f$ electrons are below the fermi level), which explains the non-heavy-fermion and non-valence fluctuation in $\mathrm{YbCoGa}_{5}$. Generally, the DOS at Fermi level $N\left(E_{F}\right)$ mainly consisting of Co-3d and Ga- $4 p$ states is the origin of the similarity between $\mathrm{YCoGa}_{5}, \mathrm{YbCoGa}_{5}$ and LuCoGa 5 . In contrast, $\mathrm{Pu} 5 f$ electrons play an important role in $\mathrm{PuCoGa}_{5}$, which confirms that the f-electrons and its magnetism is related to the superconductivity in 


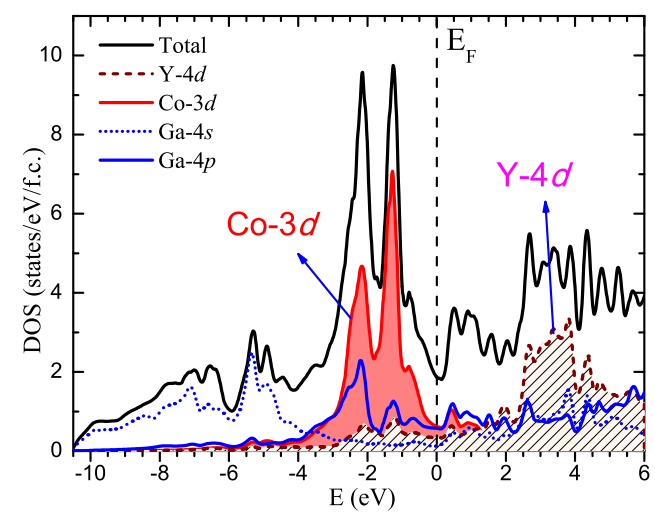

Figure 5: The calculated total and projected density of states (DOS) of YCoGa, where the partial density of states of Y-4d, and Co-3d are also shown as filled color.

$\mathrm{PuCoGa}_{5}$.

\section{Conclusions}

High quality single crystals of $\mathrm{YCoGa}_{5}$ were obtained from the Ga selfflux method. The lattice parameters, fractional coordinates, and thermal parameters of YCoGa 5 were determined from single-crystal X-ray diffraction measurements. The electrical resistivity and specific heat were measured, indicating that $\mathrm{YCoGa}_{5}$ is a metal with similar physical properties to $\mathrm{YbCoGa}_{5}$ and $\mathrm{LuCoGa}_{5}$. The band structure calculation results demonstrate that its Fermi level mainly consist of Co- $3 d$ and Ga- $4 p$ states, which is as same as $\mathrm{YbCoGa}_{5}$ and $\mathrm{LuCoGa}_{5}$.

\section{Acknowledgements}

We are very grateful to Dr. Lei Zhang for the help on Powder X-ray experiments. Work at High magnetic field lab (Hefei) was supported by the State Key Project of Fundamental Research, China (2010CB923403), National Basic Research Program of China (973 Program), No. 2011CBA00111, and National Natural Science Foundation of China (Grants No. 11204312). Work at Brookhaven is supported by the US DOE under Contract No. DEAC02-98CH10886. 


\section{Supplementary data}

Crystallographic information file for $\mathrm{YCoGa}_{5}$.

Figure: Band dispersions in $\mathrm{YCoGa}_{5}$ along high-symmetry directions. The three bands cross Fermi level are shown in color lines.

\section{References}

\section{References}

[1] C. Petrovic, P.G. Pagliuso, M.F. Hundley, R. Movshovich, J.L. Sarrao, J.D. Thompson, Z. Fisk, P. Monthoux, J. Phys.: Condens. Matter 13 (2001) L337-L342.

[2] J.L. Sarrao, L.A. Morales, J.D. Thompson, B.L. Scott, G.R. Stewart, F. Wastin, J. Rebizant, P. Boulet, E. Colineau, G.H. Lander, Nature(London) 420 (2002) 297-299.

[3] Michael R. Norman, Science 332 (2011) 196-200.

[4] C. Petrovic, R. Movshovich, M. Jaime, P.G. Pagliuso, M.F. Hundley, J.L. Sarrao, Z. Fisk, J.D. Thompson, Europhys. Lett. 53 (2001) 354359.

[5] H. Hegger, C. Petrovic, E.G. Moshopoulou, M.F. Hundley, J.L. Sarrao, Z. Fisk, and J.D. Thompson, Phys. Rev. Lett. 84 (2000) 4986-4989.

[6] J. Hudis, R. Hu, C.L. Broholm, V.F. Mitrović, C. Petrovic, J. Magn. Magn. Mater. 307 (2006) 301-307.

[7] Y. Ishikawa, D. Kato, A. Mitsuda, T. Mizushima, T. Kuwai, J. Magn. Magn. Mater. 635 (2004) 272-276.

[8] Yu.N. Grin, Ya.P. Yarmolyuk, E.I. Gladyshevskii, Sov. Phys. Crystallogr. 24 (1979) 137.

[9] E.K. Okudzeto, K. Kuga, S. Nakatsuji, J.Y. Chan, Crystal Growth \& Design 9 (2009) 1956-1959.

[10] T.D. Matsuda, Y. Haga, H. Sakai, D. Aoki, S. Ikeda, E. Yamamoto, H. Shishido, R. Settai, H. Harima, Y. Onuki, J. Phys. Soc. Japan 77 (2008) 024704-024709. 
[11] S. Watanabe, K. Miyake, J. Phys.: Condens. Matter. 23 (2011) 094217094227.

[12] J. M. Lawrence, P. S. Riseborough, R. D. Parks, Rep. Prog. Phys. 44 (1981) 1-81.

[13] K. Ishida, K. Okamoto, Y. Kawasaki, Y. Kitaoka, O. Trovarelli, C. Geibel, F. Steglich, Phys. Rev. Lett. 89 (2002) 107202-107205.

[14] D.T. Adroja, S.K. Malik, B.D. Padalia, S.N. Bhatia, R. Walia, R. Vijayaraghavan, Phys. Rev. B 42 (1990) 2700-2703.

[15] U. Subbarao, S.C. Peter, Inorg. Chem. 51 (2012) 6326-6332.

[16] R. E. Majewski, A. S. Edelstein, A. T. Aldred, A. E. Dwight, J. Appl. Phys. 49 (1978) 2096-2097.

[17] P. Bordet, J.L. Hodeau, P. Wolfers, G. Krill, F. Weiss, M. Marezio, J. Magn. Magn. Mater. 63-64 (1987) 524-526.

[18] N.D. Mathur, F.M. Grosche, S.R. Julian, I.R. Walker, D.M. Freye, R.K.W. Haselwimmer, G.G. Lonzarich, Nature 394 (1998) 39-43.

[19] E. Svanidze, E. Morosan, Phys. Rev. B 85 (2012) 174514-174518.

[20] G.M. Sheldrick, In SHELXL-97, Program for Refinement of Crystal Structures, University of Göttingen: Göttingen, Germany, 1997.

[21] P.E. Blöchl, Phys. Rev. B 50 (1994) 17953-17979.

[22] M. Torrent, F. Jollet, F. Bottin, G. Zérah, X. Gonze, Comp. Mater. Sci. 42 (2008) 337-351.

[23] X. Gonze, B. Amadon, E.A. Anglade, Comput. Phys. Comm. 180 (2009) 2582-2615.

[24] X. Gonze, J.M. Beuken, R. Caracas, F. Detraux, M. Fuchs, G.M. Rignanese, L. Sindic, M. Verstraete, G. Zerah, F. Jollet, M. Torrent, A. Roy, M. Mikami, P. Ghosez, J.Y. Raty, D. Allan, Comp. Mater. Sci. 25 (2002) 478-492.

[25] X. Gonze, Z. Kristallogr. 220 (2005) 558-562. 
[26] J.P. Perdew, K. Burke, M. Ernzerhof, Phys. Rev. Lett. 77 (1996) 38653868.

[27] H.J. Monkhorst, J.D. Pack, Phys. Rev. B 13 (1976) 5188-5192.

[28] Z. Kletowski, R. Fabrowski, P. Slawifiski, Z. Henkie, J. Magn. Magn. Mater. 166 (1997) 361-364. 